ISSN: 2600-5859

\title{
Plan de comunicación para la fábrica Costurìn de la cuidad Cuenca-Ecuador
}

\section{Communication plan for the factory Costurin de la ciudad Cuenca-Ecuador}

Kléber Antonio Luna Altamirano. ${ }^{1}$, Adriana Edelmira Aguilar Patiño. ${ }^{2}$, Jessica Esperanza Sicha Suconota. ${ }^{3} \&$ Jessica Gabriela Tenecela Pillco. ${ }^{4}$

Recibido: 11-12-2019 / Revisado: 19-12-2019 / Aceptado: 22-12-2019 / Publicado: 03-01-2020

\section{Resumen DOI: https://doi.org/10.33262/concienciadigital.v3i1.1113}

La Empresa textil Costurin, se encuentra ubicada en la ciudad de Cuenca-Ecuador, considerada una fábrica de textil de gran importancia debido a su nivel de producción para ofertar sus productos dentro del mercado comercial desde el año 2014 hasta la presente fecha. El problema de la presente investigación radica en la escasa comunicación interna en el área de producción de la fábrica, por la inadecuada gestión y coordinación de los directivos al momento de orientar la realización de las actividades, el cual ocasiona atrasos en el cumplimiento de metas establecidas. El objetivo del presente estudio es diseñar un plan de comunicación para el clima organizacional, que respalde el poder motivar a los empleados a que realicen sus actividades de producción de la mejor forma, establecer un clima adecuado para la fábrica, obteniendo mejores resultados de producción y logrando una estabilidad laboral al personal. La metodología del estudio es de tipo descriptiva con un enfoque cuantitativo, el cual se utilizará el método deductivo, se aplican encuestas dirigidas al personal, quienes aportaron la información necesaria para el cumplimiento del

${ }^{1}$ Doctor (c) en Ciencias Sociales Mención Gerencia Universidad del Zulia República Bolivariana de Venezuela, Profesor Investigador Unidad Académica de Administración Universidad Católica de Cuenca, E-mail: klunaa@ucacue.edu.ec

${ }^{2}$ Estudiante de la carrera de Contabilidad y Auditoría en la Unidad Académica de Administración de la Universidad Católica de Cuenca, E-mail: adriana.aguilar70@est.ucacue.edu.ec

${ }^{3}$ Estudiante de la carrera de Contabilidad y Auditoría en la Unidad Académica de Administración de la Universidad Católica de Cuenca, E-mail: jessica.sicha.71@est.ucacue.edu.ec

${ }^{4}$ Estudiante de la carrera de Contabilidad y Auditoría en la Unidad Académica de Administración de la Universidad Católica de Cuenca, E-mail: jessica.tenecela.69@est.ucacue.edu.ec 
objetivo planteado. Los resultados proporcionan que, al aumentar la comunicación dentro del clima organizacional traerá beneficios tales como: personal motivado y organizado para realizar mejor su actividad, facilita la comunicación con su entorno obteniendo los resultados requeridos de producción.

Palabras clave: Plan de comunicación, clima organizacional, nivel de producción, motivación al personal.

\section{Abstract}

The Costurin Textile Company is located in the city of Cuenca-Ecuador, considered a textile factory of great importance due to its level of production to offer its textile products within the commercial market from 2014 to the present date; The problem of the present investigation lies in the demotivation of the employees, high turnover of the employees, slow production, reduced kindness to communicate, diminished confidence to communicate their concerns, loss of confidence to share confident information and the misunderstandings that usually arise from the wrong communication, it allows that there is no adequate communication. The objective of this study is to design a communication plan for the organizational climate, which supports the ability to motivate employees to carry out their production activities in the best way, establish a suitable climate for the factory obtaining better production results and giving job stability to staff. The methodology of the study is of a descriptive type with a quantitative approach, which will use the deductive method, surveys directed to the personnel are applied, and who provided the necessary information for the fulfillment of the previously stated objective. The results provide that increasing communication within the organizational climate will bring us benefits such as: motivated and organized personnel to better perform their activity, facilitates communication with their environment, obtaining the required production results.

Keywords: Communication plan, organizational climate, production level, motivation to staff.

\section{Introducción}

En la ciudad de Cuenca Ecuador, la Fábrica de Costuras Internacionales Cía. Ltda. es una de las empresas creada por la cadena de la Corporación “Gerardo Ortiz \& Hijos" fue fundada el 21 de 
octubre del 2014, cuenta con sucursales alrededor del Ecuador, en las ciudades de Quito, Ambato, Manta y Guayaquil, su actividad principal es la fabricación de artículos textiles tales como: edredones, sabanas, toallas, alfombras, entre otros, siendo una de las empresas más importantes en el Ecuador, reconocida por su oferta de productos de primera calidad que cubre la demanda de los consumidores a nivel nacional.

El problema de la investigación, se presenta por la escasa comunicación dentro del área de producción, en el que se observa una reducida amabilidad para comunicar, poca confianza para comunicar sus inquietudes, pérdida de confianza por compartir información confidente y los malos entendidos que suelen surgir por la falta de fluidez en la comunicación, el mismo permite que esta sea inadecuada. Narváez (2011) afirma:

La comunicación dentro de cualquier empresa debe ser consistente, permanente y oportuna, logrando para así lograr una dirección y orientación clara de las actividades de la organización; alcanzando la coordinación e integración con cada departamento y elemento humano que labora dentro de la empresa, brindando así un mejor producto o servicio para los clientes (p.12)

La poca comunicación dentro del clima organizacional es un fenómeno que se presenta en gran medida dentro de los ambientes laborales, dando origen a desacuerdos que se desencadena por la disminuida comunicación y organización afectando al desenvolvimiento y agilidad al momento de ejecutar su actividad de producción.

El objetivo del presente estudio es diseñar un plan de comunicación para el clima organizacional, respalde el poder motivar a los empleados a que realicen sus actividades de producción de la mejor manera, establecer un clima adecuado para la fábrica obteniendo resultados favorables dentro de la producción. Charry (2018) afirma:

El clima organizacional o laboral es el constructo intangible que se relaciona a la percepción que poseen los trabajadores sobre su entorno laboral. Es el tono emocional interno de los empleados de la entidad con respecto a distintos factores en un momento dado (p.28).

La presente investigación es de tipo descriptiva, pues se quiere indicar propiedades y características del fenómeno a analizar, para ello se recaba información y se describe tendencias de grupos o poblaciones (Hernández, Fernández y Baptista, 2014). El enfoque del estudio es cuantitativo pues 
se busca comprobar la hipótesis apoyándose en la medición numérica y el análisis estadístico (Hernández, Fernández y Baptista, 2014).

Se utiliza el método deductivo pues se realiza una construcción del conocimiento que parte de lo general a lo particular y deduce la información recopilada, Bernal (2010) afirma:

Este método de razonamiento consiste tomar conclusiones generales para obtener explicaciones particulares. El método se inicia con el análisis de los postulados, teoremas, leyes, principios, (...), de aplicación universal y de comprobada validez, para aplicarlos a soluciones o hechos particulares (p.59).

Se maneja como instrumento de investigación la encuesta, la cual permite extraer datos para formar bases de información real y conocer las falencias del fenómeno estudiado.

Es una técnica propia del diseño de investigación de campo. Generalmente la encuesta se utiliza para indagar, averiguar opiniones, mediante preguntas estructuradas en base a un proceso metódico como es la operacionalización de las variables. Al formular estas interrogantes sobre tal o cual fenómeno investigado permite que las respuestas tengan objetividad (Cabezas, Andrade y Torres, 2018, p.81).

La presente investigación está conformada por tres partes fundamentales en la cual intervienen los siguientes: se puntualiza el estado del arte como primer punto, en el cual la información contenida está basada en la opinión de varios autores de artículos científicos que aportan y sostienen con su conocimiento al presente estudio, seguidamente se puntualiza la metodología, en el describe el plan de comunicación que recomienda aplicar estrategias para dar paso a una efectiva comunicación, en conclusión se alcanzara a reducir factores negativos que afecta a un buena comunicación, dando paso al cumplimiento de las metas establecidas en el área de producción.

\section{Estado del arte}

El diseñar un plan de comunicación para mejorar el clima organizacional para la Empresa Textil Costuras Internacionales (Costurin) ubicada en la ciudad de Cuenca-Ecuador, es de suma importancia ya que este permitirá conocer la causa, motivación, desempeño, satisfacción y posibles soluciones para los trabajadores que laboran en la organización empresarial, por ello algunos autores contribuyen con sus investigaciones relacionados con el tema del presente estudio, entre ellos: Monserrat (2008) explica que para la expansión y el crecimiento de la empresa se examinará la contribución de las estrategias de comunicación, además de esto se estudiara un análisis de las 
estrategias ya plateados con anterioridad para una comunicación más amplia y concreta en la empresa.

Oyarvide, Reyes y Montaño (2017) interpretan que la comunicación interna posibilita obtener mayor aptitud, fomentar el sentimiento de pertenencia, establecer a los empleados en una efectiva cultura colectiva, conservar a los excelentes y así conseguir el éxito empresarial. La dirección y los diferentes departamentos de la empresa es superior cuando los trabajadores están bien informados, cuando conocen la misión a donde quieren llegar, los valores, la estrategia empresarial y si existe una línea eficaz de comunicación entre los empleados dentro del área laboral.

Moreno (2012) propone un plan de comunicación, este propicia explicar a términos expansivos la planeación de la organización de la empresa, las mismas que establece las vías a medio plazo para obtenerlos mediante la descripción del posicionamiento e imagen de la misma, la planificación metodológica se aplica para las cambiantes oportunidades del mercado las mismas que establece un ajuste variable entre los objetivos y recursos de la empresa, este factor es sumamente importante con un punto de partida para la implementación de un sistema de comunicación integral es planeación estratégica de la organización en dicha empresa, el objetivo de esta planificación consiste en modelar y ordenar las diferentes áreas del negocio para satisfacer el crecimiento y el beneficio de la empresa.

Bravo, González y Duque (2007) plantean una diversidad de planificaciones que facilitara el perfeccionamiento de las capacidades de los empleados, motivándoles y dando conocimiento y beneficios para resolver el problema antes mencionada, dando así resultados de la conclusión de clima organizacional de dicha empresa, así como también algunas apariencias que afectan en el estímulo y conducta de los ayudantes reconocidos de forma autónomo al mecanismo de cálculo del clima. Gonzalvéz (2010) aporta con su investigación, en todo libro que define la comunicación empresarial, se puede decir que existe muy alto el interés por parte de los profesionales, que es conocido también como la gestión integral; esta disciplina es sumamente importante por ende no se puede obviar.

Páez y Rodríguez (2004) proponen evidenciar o demostrar el desarrollo del plan de comunicación que se elaborará en esta empresa, el mismo que contendrá una serie de elaboración de los mensajes de campaña educativa para aumentar el consumo de frijoles en una comunidad urbana. Naranjo (2013) sugiere diseñar un plan de comunicación que facilite a implementar acciones con relación 
al ordenamiento entre los estudiantes de la UNAC, con la finalidad de alcanzar sus metas y objetivos estratégicos. El mismo autor plantea una estructura en el cual se puede analizar la visión de la gestión de la comunicación por mecanismos, ámbitos o espacios de actuación, de esta manera permitirá visualizar de forma global todos los procesos de un ente o departamento.

Por su parte los autores como: Cotton (s.f.) aporta con su investigación con el propósito de manifestar proyectos organizacionales del desarrollo debido a que es una práctica frecuente, centrarse en los indicios relacionados con los niveles jerárquicos más bajos y enfocarse en plantear las mejoras para la empresa. Rivera, Rojas, Ramírez y Alvares (2005) aportan con su definición que la alta gerencia debe transmitir la información adecuada e indispensable para capacitar, estimulando a las personas que cumplen con su labor para que se movilicen hacía los resultados habituales y personales, eliminando así todas las falencias e interferencias o malos entendidos, así como también creando espacios para la retroalimentación de una buena comunicación.

Estrada y Restrepo (2010) en su investigación explica que, para estar entrelazados con el entorno cambiante y dinámico, las organizaciones necesitan fortalecer su modelo de comunicación ya que de esta manera se puede articular sistemáticamente los procesos y sostener o dar mantenimiento a la comunicación adecuada en el tiempo.

Charry (2018) con su aporte explica que en toda entidad pública la gestión de comunicación debe manifestar al mundo contemporáneo y competitivo con un alto nivel de comunicación ordenada e interactiva, experto de escuchar a los participantes examinando transferir igualdad, percepciones que estimulen a informar y vincularse. Para minimizar la comunicación informal y el rumor, la comunicación oficial debe ser siempre la primera en llegar a los trabajadores ya que es muy importante para que haya una comunicación eficaz entre los funcionarios. El mismo autor explica que la comunicación interna debe producirse con una anticipada planificación, para lo dicho se debe tener objetivos y resultados concretos y transparentes. Esta comunicación debe ser una herramienta organizativa sumamente importante así logrará ser más efectiva y eficiente.

El aporte de los autores mencionados es de gran utilidad para el correcto diseño del plan de comunicación en la empresa, ya que de esta manera se analiza las causas que originan el problema, con el propósito de poder contrarrestar el mismo, con la contribución de los investigadores mencionados se puede considerar los puntos negativos en diferentes áreas laborales, logrando estimular la correcta y adecuada comunicación interna. 
ISSN: 2600-5859

\section{Metodología}

La investigación que se presenta es de tipo descriptiva, con un enfoque cuantitativo, en el cual se utilizara el método deductivo, se aplican encuestas dirigidas al personal para obtener la información para el posterior análisis, las mismas que fueron aplicadas a 105 personas dentro de las cuales está incluido personal administrativo y operativo que laboran en la fábrica de Costurin de la ciudad de Cuenca-Ecuador, considerados como universo, este mismo número de encuestados, se les consideró como muestra, con el propósito de no sesgar los datos y que el contenido de la información sea más real. A continuación, se detalla los cargos del personal a quienes fueron encuestados.

Tabla 1. Participación del personal de la fábrica Costurin

\begin{tabular}{lc}
\hline Personal total & Numero \\
\hline Gerente General & 1 \\
Gerente Planta & 1 \\
Secretarias & 2 \\
Supervisora & 1 \\
Jefe de despacho & 1 \\
Líderes de planta & 10 \\
Despachadores & 6 \\
Personal de Producción & 83 \\
\hline \multicolumn{1}{c}{ Total } & 105 \\
\hline
\end{tabular}

Fuente: Elaboración propia

\section{Resultados}

Se visualiza en la primera tabla, la muestra de individuos correspondientes a la fábrica de Costurin Cuenca-Ecuador, son 105 personas encuestadas, a quienes se recaudó información, las encuestas se aplicaron al personal administrativos y a nivel operativo, cuyas edades varían desde los 25 a 50 años y entre los cuales hay personal que cuentan con un amplio conocimiento y experiencia en el proceso de producción. La información más relevante, se detalla a continuación. 
Figura 1. Comunicación escasa

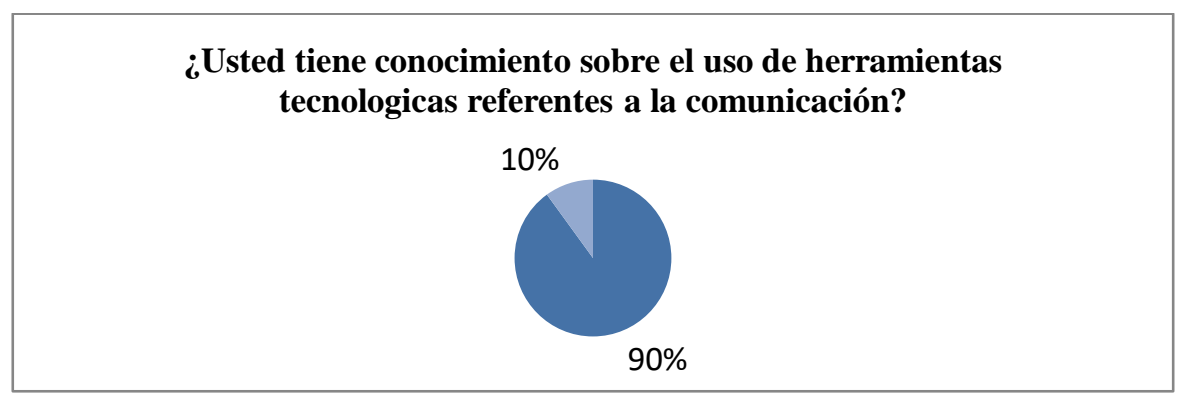

Fuente: Elaboración propia.

Como respuesta a la pregunta planteada con anterioridad se observa que el $90 \%$ del personal tiene un conocimiento escaso sobre las distintas herramientas tecnológicas que se utilizan en la actualidad, seguidamente se puede establecer una posible solución a este problema sería necesario implementar capacitaciones sobre los distintos medios tecnológicos de comunicación para garantizar que la misma sea de una forma expresiva.

Rubio y Gértrudix (2016) explican que en los últimos tiempos las diferentes aplicaciones tecnológicas se fundamentan en las necesidades de un desarrollo continuo en nuevos procesos y medios de comunicación, estas nuevas innovaciones de comunicación plantean una nueva tecnología que facilita la eficacia y fluidez de la misma

Figura 2. Comunicación interna

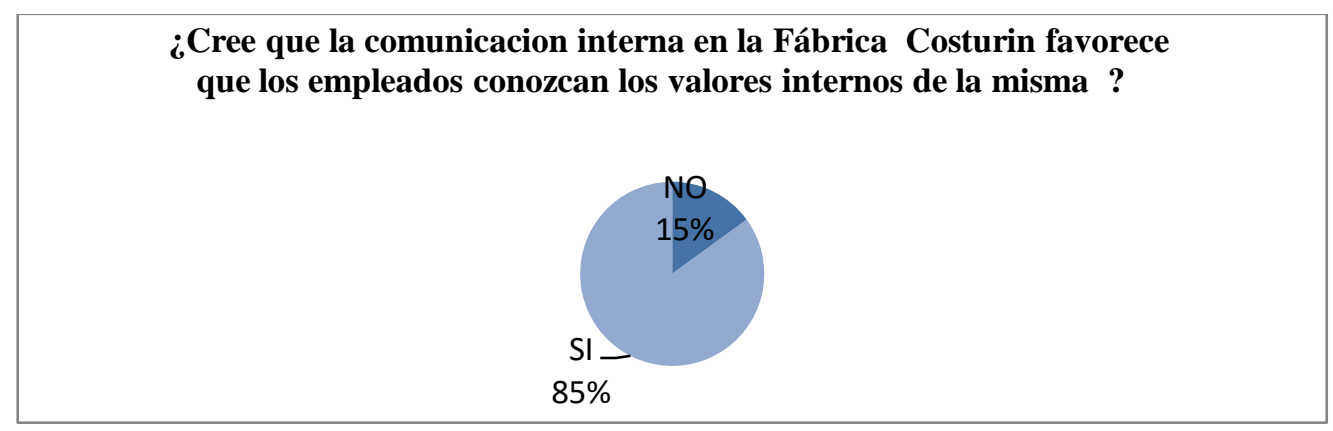

Fuente: Elaboración propia

Como resultados de la pregunta planteada se puede observar que el $85 \%$ de los trabajadores de la fábrica están en total acuerdo sobre el enunciado en cuestión motivo por el cual se considera que 
la comunicación interna es de gran valor debido que es necesario que los trabajadores conozcan valores dentro de la fábrica. Balarezo (2014) afirma:

Las organizaciones a nivel nacional en los últimos años han determinado que es importante tener en cuenta que toda comunicación se realiza en el marco de los vínculos humanos y que estos se expresan por medio de la manera en que las personas realizan su tarea, como se relacionan y complementan de acuerdo con sus propias culturas, se construye así una cultura organizacional compuesta por un conjunto de valores, creencias y comprensiones que comparten los integrantes de una organización (p.6).

Figura 3. Conocimiento de los comunicados a tiempo

\begin{tabular}{|c|c|}
\hline iA usted le comunican oportunamente las desiciones \\
tomadas en la empresa? \\
\hline
\end{tabular}

Fuente: Elaboración propia

EL 75\% del personal que labora en la fábrica no es comunicado puntalmente en lo que respecta a los avisos, posteriormente se puede visualizar que si el personal no es comunicado con un respectivo lapso de tiempo las respectivas indicaciones puede haber conflictos y retrasos en ciertas actividades a desempeñar. Capriotti (1998) sostiene: "La Comunicación interna cumple una serie de funciones que le permiten a la organización mejorar la eficacia en toda su actividad interior, lo cual redundará, sin duda, en una mayor competitividad externa de la compañía" (p.4).

Figura 4. Comunicación efectiva

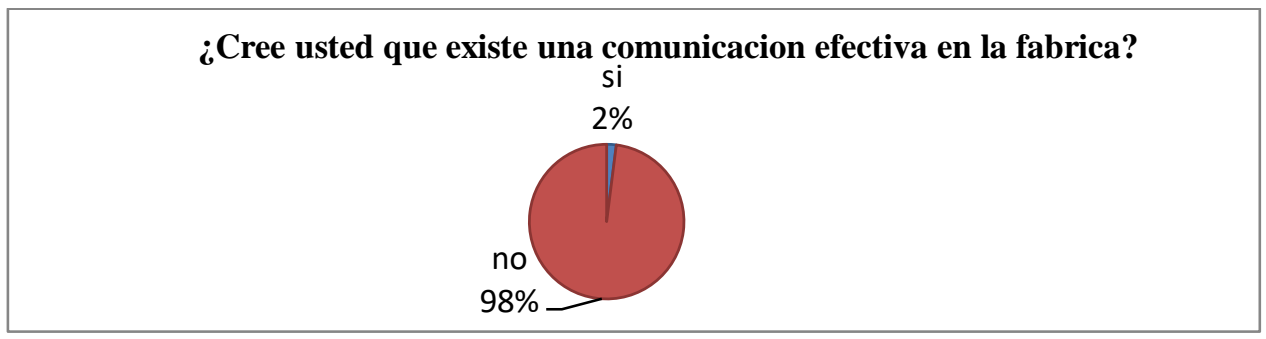

Fuente: Elaboración propia. 
En esta pregunta planteada, se puede ver con total claridad que el $98 \%$ de las personas encuestadas manifiestan que no existe una comunicación efectiva entre los funcionarios de la empresa, por lo tanto, para poder mejorar el presente problema, de debe tener en cuenta que, la persona quien transmite el mensaje lo debe hacer de forma clara, precisa, entendible y sin confusiones.

Un asunto preocupante en el proceso de comunicación acurre al no existir canales definidos en relación al tipo de comunicación que permita el intercambio personal, ya que es necesario que la persona que comunica se identifique con sentido de pertenencia cultural y a través del mensaje logre establecer una comunicación efectiva, bien sea en su entorno familiar, social, organizacional o empresarial. (Quero, Mendoza y Torres, 2014, p.23).

Figura 5. Plan de comunicación

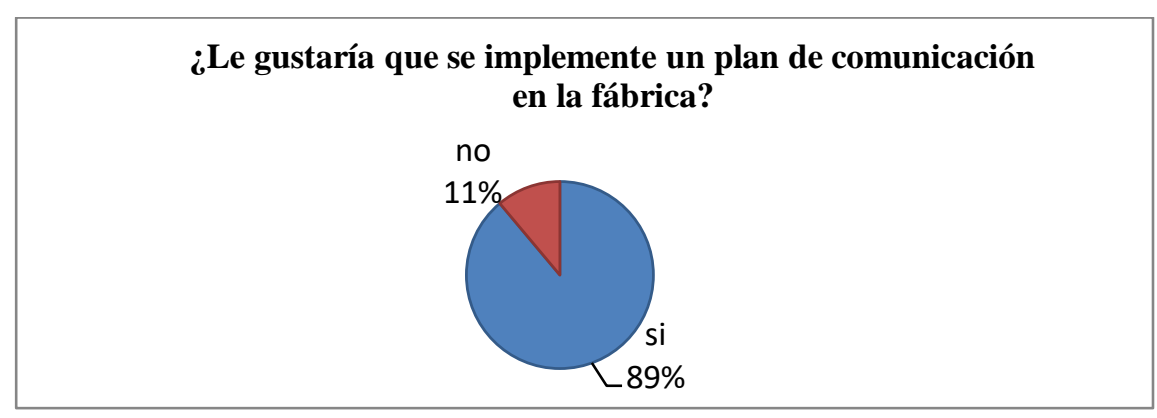

Fuente: Elaboración propia

Se puede visualizar que un $89 \%$ está de acuerdo que se implemente un plan de comunicación, esto ayudara a considerar diferentes puntos negativos que se da en la fábrica Costurin, de esta manera se podrá combatir con algunas consecuencias que se presentaría en un futuro. Capriotti (1998) sostiene:

Esta perspectiva es mucho más comprometida y más difícil que la anterior, e implica reconocer la importancia de la comunicación directa y personal entre los miembros de la organización, y la participación de los empleados en las sugerencias y en la puesta en marcha de las iniciativas comunicativas y de relación. (p.1)

Para dar fuerza a la presente investigación se realiza el análisis del estudio FODA, mismo que está estructurado en base a las preguntas de la misma encuesta realiza anteriormente, partiendo de ello se establecen dos factores, internos y externos con relación al problema planteado. 
ISSN: 2600-5859

Tabla 2. Matriz FODA Factores Internos

\begin{tabular}{ll}
\hline Fortalezas & Debilidades \\
\hline -Control del proceso de producción & -Baja coordinación en el cumplimiento de los programas \\
& establecidos \\
- Muy buena publicidad & -Existe apatía con el personal \\
-Buena logística (transporte de productos) & -Poco compañerismo \\
-Amplia Capacidad de Inversión & -Escaza fluidez de la comunicación \\
-Existencia de líderes por área & -No existe conocimiento de las inquietudes del personal
\end{tabular}

Fuente: Elaboración Propia

Tabla 3. Matriz FODA Factores Externos

\begin{tabular}{ll}
\hline \multicolumn{1}{c}{ Oportunidades } & \multicolumn{1}{c}{ Amenazas } \\
\hline -Ampliar la publicidad de los productos textiles a nivel internacional & $\begin{array}{c}\text {-Copia de las estrategias de publicidad, } \\
\text { por parte de la competencia }\end{array}$ \\
-Implementar capacitaciones de comunicación & -Nuevos mercados \\
-Aprovechar las nuevas tecnologías para utilizar en el manejo de la & -Ventas online \\
comunicación adecuada & \\
-Existe gran aceptación en el mercado comercial de los productos & -Identificación de nuevos consumidores \\
fabricados & \\
\hline
\end{tabular}

\section{Fuente: Elaboración Propia}

Estructurada la matriz FODA, los factores tanto internos como externos, apoyan al diseño de un modelo de plan de comunicación, este análisis presenta la realidad actual de la empresa con relación a la comunicación interna, cada una de las variables permitirá tomar decisiones estratégicas pues éste será el camino que permite ver la realidad del ambiente interno de la organización.

A continuación, se presenta el modelo de plan de comunicación para la fábrica Costurin, éste se diseñó a partir de la necesidad institucional, la información obtenida a través de encuestas, representan un alto valor para la estructura de este plan, con ello se logrará que mejorar el clima organizacional por medio de una vía más eficiente de comunicación. 
ISSN: 2600-5859

WWW.concienciadigital.org

Vol. 3, $\mathrm{N}^{\circ} 1$, p. 75-90, enero - marzo, 2020

Tabla 4. Plan de Comunicación

\begin{tabular}{|c|c|c|c|c|c|c|}
\hline $\begin{array}{c}\text { Problema } \\
\text { Primordial }\end{array}$ & Objetivo & Estrategias & Responsable & $\begin{array}{c}\text { Fecha del } \\
\text { proceso }\end{array}$ & $\begin{array}{l}\text { Actividades de } \\
\text { gestión de } \\
\text { control }\end{array}$ & Resultados \\
\hline $\begin{array}{l}\text { Desorden por la } \\
\text { comunicación } \\
\text { ineficaz }\end{array}$ & $\begin{array}{c}\text { Tratar de } \\
\text { establecer reglas y } \\
\text { valores dentro de } \\
\text { la fabrica }\end{array}$ & $\begin{array}{c}\text { Incorporación } \\
\text { de un buzón de } \\
\text { sugerencias } \\
\text { dirigida hacia } \\
\text { los empleados }\end{array}$ & $\begin{array}{c}\text { Área de } \\
\text { Marketing }\end{array}$ & Renovación anual & $\begin{array}{c}\text { Revisar el buzón } \\
\text { de una forma } \\
\text { trimestral }\end{array}$ & $\begin{array}{c}\text { Crear una mejor } \\
\text { organización en } \\
\text { los empleados }\end{array}$ \\
\hline $\begin{array}{c}\text { Poco } \\
\text { compañerismo }\end{array}$ & $\begin{array}{c}\text { Compañerismo } \\
\text { adecuado entre los } \\
\text { empleados }\end{array}$ & $\begin{array}{c}\text { Programas de } \\
\text { integración } \\
\text { social }\end{array}$ & $\begin{array}{c}\text { Líderes y } \\
\text { supervisor de la } \\
\text { fábrica }\end{array}$ & Diariamente & $\begin{array}{c}\text { Charlas } \\
\text { psicosociales }\end{array}$ & $\begin{array}{c}\text { Buen } \\
\text { compañerismo }\end{array}$ \\
\hline $\begin{array}{c}\text { Escasa } \\
\text { comunicación }\end{array}$ & $\begin{array}{l}\text { Comunicación } \\
\text { apropiada entre } \\
\text { directivos y } \\
\text { funcionarios }\end{array}$ & $\begin{array}{l}\text { Capacitación } \\
\text { presencial y } \\
\text { tecnológica }\end{array}$ & $\begin{array}{l}\text { Ingenieros, } \\
\text { supervisores, } \\
\text { líderes y } \\
\text { empleados }\end{array}$ & Diariamente & $\begin{array}{c}\text { Capacitación } \\
\text { semestral }\end{array}$ & $\begin{array}{c}\text { Comunicación } \\
\text { confortable entre } \\
\text { el equipo de } \\
\text { trabajo de } \\
\text { Costurin }\end{array}$ \\
\hline $\begin{array}{c}\text { Poco espacio } \\
\text { adecuado para el } \\
\text { área de trabajo }\end{array}$ & $\begin{array}{c}\text { Espacio eficaz en } \\
\text { las distintas áreas } \\
\text { laborales }\end{array}$ & $\begin{array}{c}\text { Oficio al } \\
\text { gerente general } \\
\text { del grupo Ortiz } \\
\& \text { hijos }\end{array}$ & $\begin{array}{c}\text { Gerente de la } \\
\text { fabrica }\end{array}$ & Inicio del año & - & $\begin{array}{c}\text { Lugar seguro, } \\
\text { amplio, sano y } \\
\text { confortable }\end{array}$ \\
\hline $\begin{array}{c}\text { Equipos de } \\
\text { comunicación en } \\
\text { mal estado }\end{array}$ & $\begin{array}{l}\text { Equipos nuevos } \\
\text { con un buen } \\
\text { funcionamiento }\end{array}$ & $\begin{array}{c}\text { Oficio al } \\
\text { gerente general } \\
\text { del grupo Ortiz } \\
\& \text { hijos }\end{array}$ & $\begin{array}{c}\text { Gerente de la } \\
\text { fábrica }\end{array}$ & $\begin{array}{c}\text { Mantenimiento } \\
\text { cada año }\end{array}$ & $\begin{array}{c}\text { Supervisar } \\
\text { mensualmente }\end{array}$ & $\begin{array}{c}\text { Comunicación } \\
\text { oportuna e } \\
\text { inmediata }\end{array}$ \\
\hline $\begin{array}{c}\text { Insuficiencia } \\
\text { capacitaciones } \\
\text { en la } \\
\text { comunicación }\end{array}$ & $\begin{array}{c}\text { Que tengan un } \\
\text { conocimiento } \\
\text { sobre la } \\
\text { comunicación en } \\
\text { su labor y de su } \\
\text { entorno }\end{array}$ & $\begin{array}{l}\text { Instrucciones } \\
\text { para ser un } \\
\text { mejor líder o } \\
\text { empleado } \\
\text { mediante la } \\
\text { comunicación }\end{array}$ & $\begin{array}{c}\text { Ingenieros, } \\
\text { supervisores, } \\
\text { líderes y } \\
\text { empleados }\end{array}$ & Cada tres meses & $\begin{array}{c}\text { Ilustraciones } \\
\text { semestrales }\end{array}$ & $\begin{array}{l}\text { Realización de } \\
\text { sus actividades } \\
\text { con máxima } \\
\text { potencial } \\
\text { mediante la } \\
\text { comunicación }\end{array}$ \\
\hline $\begin{array}{c}\text { Carencia de } \\
\text { comunicación } \\
\text { por la tecnología } \\
\text { (teléfonos) }\end{array}$ & $\begin{array}{c}\text { Formular reglas } \\
\text { para la } \\
\text { prohibición de } \\
\text { teléfonos en el } \\
\text { momento de } \\
\text { comunicar }\end{array}$ & $\begin{array}{c}\text { Reuniones en } \\
\text { donde hagan } \\
\text { conocer dichas } \\
\text { reglas }\end{array}$ & $\begin{array}{c}\text { Gerente de la } \\
\text { fabrica }\end{array}$ & Trimestral & $\begin{array}{c}\text { Estimular al } \\
\text { personal para } \\
\text { tener una } \\
\text { comunicación } \\
\text { eficaz sin } \\
\text { interrupciones }\end{array}$ & $\begin{array}{c}\text { Laborar teniendo } \\
\text { en cuenta las } \\
\text { reglas y así se } \\
\text { logrará una } \\
\text { buena } \\
\text { comunicación }\end{array}$ \\
\hline
\end{tabular}

Fuente: Elaboración propia 
En el presente plan se evidencia cambios positivos para una correcta comunicación, en el cual se observan puntos importantes para la mejora interna de la fábrica Costurin. Se puede recalcar que el propósito es crear una comunicación interna fluida y eficaz, logrando un mejor compañerismo entre los funcionarios de las diferentes áreas laborales. Para lograr esta meta se lo realizará a través de:

- Mayor interés en la comunicación mediante capacitaciones.

- Equipos de comunicación en óptimas condiciones.

- Funcionarios dispuestos al cambio.

\section{Conclusiones}

El diseñar un plan de Comunicación para el mejoramiento del clima organizacional para la fábrica Costurin de la ciudad de Cuenca-Ecuador, permite visualizar el fenómeno estudiado y resolver la problemática que se presenta por medio de las estrategias trazadas. Este estudio pretende buscar la vía adecuada para una mejora en la comunicación interna de la empresa, con ello se tratará de mejorar los procesos administrativos y de producción, ya que este se ve afectado por una ruptura de la comunicación, los directivos con esta investigación podrán tomar los mejores correctivos a través de una correcta toma de decisiones.

Este plan, permite garantizar una adecuada actividad laboral, mejorar la sostenibilidad de la empresa en el mercado actual. Dentro de las metas planteadas están motivar a todos los funcionarios hacia la mejora continua, proyectando a obtener ventajas competitivas, reforzando las debilidades y manteniendo las fortalezas, con el propósito de lograr llegar al éxito empresarial. La presente investigación, aporta de manera sustancial a la rectificación de los procesos comunicativos dentro de la empresa, su impacto a corto plazo permitirá que la alta gerencia tenga una visión más acertada en los cambios necesarios para fortalecer la comunicación interna tratando de mantener los ideales institucionales. 
ISSN: 2600-5859

\section{Referencias Bibliográficas}

Bernal, C. (2010). Metodología de la investigación administración, economía, humanidades y ciencias sociales. Colombia: Pearson educación. Recuperado de http://abacoenred.com/wp-content/uploads/2019/02/El-proyecto-deinvestigaci\%C3\%B3n-F.G.-Arias-2012-pdf.pdf

Bravo, A., González, G., y Duque, J. (2017). Diseño de estrategias de mejoramiento a partir del diagnóstico de clima organizacional en una empresa distribuidora de combustibles y alimentos. Ciencias sociales aplicadas, 14(1), 1https://www.redalyc.org/jatsRepo/2654/265457559002/index.html

Cabezas, E., Naranjo, D., y Torres, J. (2018). Introducción a la metodología de la investigación científica. Sangolquí, Ecuador: Comisión Editorial de la Universidad de las Fuerzas Armadas ESPE. Recuperado de http://repositorio.espe.edu.ec/jspui/bitstream/21000/15424/1/Introduccion\%20a\%20la\%20 Metodologia\%20de\%201a\%20investigacion\%20cientifica.pdf

Cotton, P. (s.f.), Desarrollando un clima organizacional óptimo, Grandes Pymes, recuperado de https://www.grandespymes.com.ar/2017/08/26/desarrollando-un- clima- organizacionaloptimol

Charry, H. (2018). La gestión de la comunicación interna y el clima organizacional en el sector público. Comunicación, 9(1), 25-34. Recuperado de http://www.scielo.org.pe/pdf/comunica/v9n1/a03v9n1.pdf

Estrada, S., y Restrepo, L. (2010). Modelo de comunicación para organizaciones cambiantes. Scientia Et Technica, 16(44), 72-77. Recuperado de http://www.redalyc.org/articulo.oa?id=84917316013

Formanchuk, A. (2006). Comunicación Interna, Externa e Imagen Corporativa: Nuevos Paradigmas para una Economía Global. Razón y Palabra, (49), 1-5. Recuperado de http://www.redalyc.org/articulo.oa?id=199520713024

Gonzálvez, J. (2012). La cara interna de la comunicación en la empresa. Revista de Comunicación de la seeci, (28), 9-9. Recuperado de http://www.redalyc.org/articulo.oa?id=523552846007

Hernández, R., Fernández, C., y Baptista, M. (2014). Metodología de la Investigación. México: mcgraw-hill Recuperado de http://observatorio.epacartagena.gov.co/wpcontent/uploads/2017/08/metodología-de-la-investigación-sexta- edicion.compressed.pdf

Monserrat, J. (2008). Análisis comparativo de las estrategias de Comunicación en franquicia. Latina de Comunicación Social, 11(63), 106-120. Recuperado de http://www.redalyc.org/articulo.oa?id=81912006011 
Moreno, O. (2012). Gestión integral del sistema de comunicación en las organizaciones enperfeccionamiento empresarial. Ciencia en su PC, (3), 31-43. Recuperado de http://www.redalyc.org/articulo.oa?id=18132407100

Narváez, Y. (2011) Diseño de un plan de comunicación interna para mejorar el clima organizacional en el área de tripulantes de cabina de la Aerolínea LAN Ecuador, Universidad Tecnológica Equinoccial, Ecuador, http://repositorio.ute.edu.ec/handle/123456789/10612

Nooteboom, B. (2010). La dinámica de la confianza: comunicación, acción y terceras partes. Revista de Economía Institucional, 12(23), 111-133. http://www.redalyc.org/articulo.oa?id=41915521006

Oyarvide, H., Reyes, E., y Montaño, M. (2017). La comunicación interna como herramienta indispensable de la administración de empresas. Ciencias económicas y empresariales, 3(4), 296-309. https://www.researchgate.net/publication/336009673

Páez, P., y Rodríguez, L. (2004). Plan de comunicación: un buen instrumento para que su campaña tenga éxito. Agronomía Mesoamericana, 15(3), 315-326. Recuperado de http://www.redalyc.org/articulo.oa?id=43715307

Quero, Y., Mendoza, F., y Torres, Y. (2014). Comunicación efectiva y desempeño laboral en Educación Básica. Negotium, 9(27), 22-33. Recuperado de http://www.redalyc.org/articulo.oa?id=78230409001

Rivera, A., Rojas, L., Ramírez, F., y Álvarez, T. (2005). la comunicación como herramienta de gestión organizacional. Negotium, 1(2), 32-48. Recuperado de http://www.redalyc.org/articulo.oa?id=78212103

Rojas, A. (2004). La intermediación laboral. Revista de Derecho, (22), 187-210. Recuperado de https://www.redalyc.org/articulo.oa?id=85102208

Tur, V., y Monserrat, J. (2014). El plan estratégico de comunicación. Estructura y funciones. Razón y Palabra, 88. Recuperado de http://www.redalyc.org/articulo.oa?id=19953273104

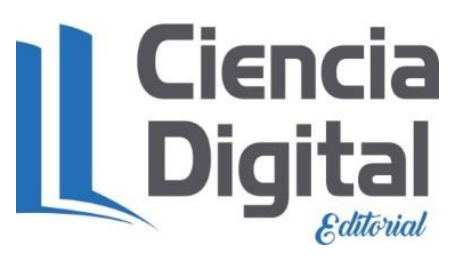




\section{Para citar el artículo indexado}

Luna Altamirano, K. A., Aguilar Patiño, A. E., Sicha Suconota, J. E., \& Tenecela Pillco, J. G. T. P. (2020). Plan de comunicación para la fábrica Costurìn de la cuidad Cuenca-Ecuador. ConcienciaDigital, 3(1), 75-90. https://doi.org/10.33262/concienciadigital.v3i1.1113

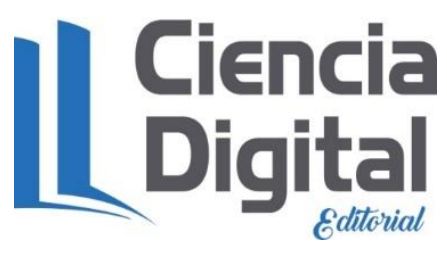

El artículo que se publica es de exclusiva responsabilidad de los autores y no necesariamente reflejan el pensamiento de la Revista Conciencia Digital.

El articulo queda en propiedad de la revista y, por tanto, su publicación parcial y/o total en otro medio tiene que ser autorizado por el director de la Revista Conciencia Digital.
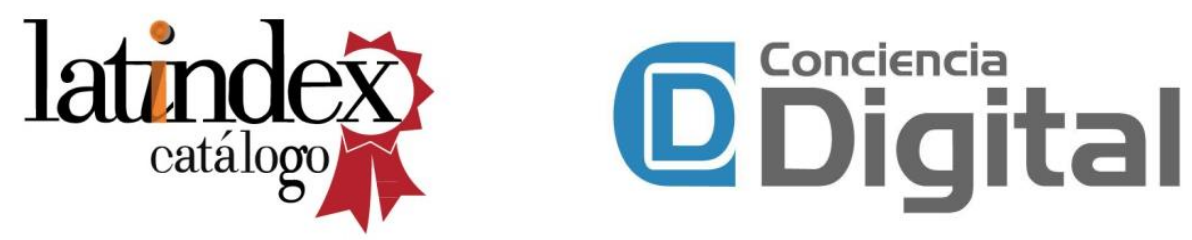